Wafi, Sammy; Wirtz, Markus A.

\title{
Visualisierungskompetenz in Deutsch und Mathematik aus Sicht von Expertinnen und Experten der Lehr-/Lernforschung und Fachdidaktik
}

Zeitschrift für interpretative Schul- und Unterrichtsforschung 4 (2015) 1, S. 119-140

Quellenangabe/ Reference:

Wafi, Sammy; Wirtz, Markus A.: Visualisierungskompetenz in Deutsch und Mathematik aus Sicht von Expertinnen und Experten der Lehr-/Lernforschung und Fachdidaktik - In: Zeitschrift für interpretative Schul- und Unterrichtsforschung 4 (2015) 1, S. 119-140 - URN: urn:nbn:de:0111-pedocs-153403 - DOI: 10.25656/01:15340

https://nbn-resolving.org/urn:nbn:de:0111-pedocs-153403

https://doi.org/10.25656/01:15340

in Kooperation mit / in cooperation with:

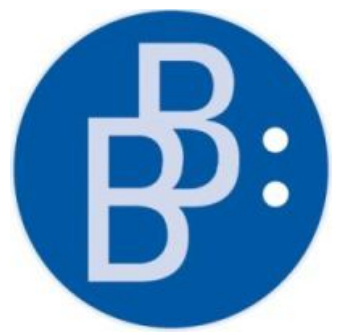

https://www.budrich.de

\section{Nutzungsbedingungen}

Gewährt wird ein nicht exklusives, nicht übertragbares, persönliches und beschränktes Recht auf Nutzung dieses Dokuments. Dieses Dokument ist ausschließlich für den persönlichen, nicht-kommerziellen Gebrauch bestimmt. Die Nutzung stellt keine Übertragung des Eigentumsrechts an diesem Dokument dar und gilt vorbehaltlich der folgenden Einschränkungen Auf sämtlichen Kopien dieses Dokuments müssen alle Urheberrechtshinweise und sonstigen Hinweise auf gesetzlichen Schutz beibehalten werden. Sie dürfen dieses Dokument nicht in irgendeiner Weise abändern, noch dürfen Sie dieses Dokument für öffentliche oder kommerzielle Zwecke vervielfältigen, öffentlich ausstellen, aufführen, vertreiben oder anderweitig nutzen.

Mit der Verwendung dieses Dokuments erkennen Sie die Nutzungsbedingungen an.

\section{Terms of use}

We grant a non-exclusive, non-transferable, individual and limited right to using this document.

This document is solely intended for your personal, non-commercial use. Use of this document does not include any transfer of property rights and it is conditional to the following limitations: All of the copies of this documents must retain all copyright information and other information regarding legal protection. You are not allowed to alter this document in any way, to copy it for public or commercial purposes, to exhibit the document in public, to perform, distribute or otherwise use the document in public.

By using this particular document, you accept the above-stated conditions of use.

\section{Kontakt / Contact:}

\section{peDOCS}

DIPF | Leibniz-Institut für Bildungsforschung und Bildungsinformation Informationszentrum (IZ) Bildung

E-Mail: pedocs@dipf.de

Internet: www.pedocs.de

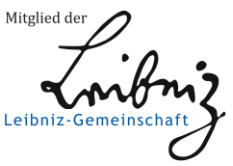




\section{Inhalt}

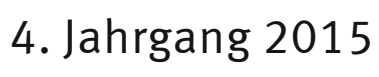

ZISU

\section{Zeitschrift für interpretative Schul- und Unterrichtsforschung}

\section{Editorial}

Ulrich Gebhard, Merle Hummrich, Kerstin Rabenstein, Sabine Reh

\section{Thementeil}

Georg Breidenstein

Thomas Grunau, Mirja Kekeritz

Matthias Martens, Barbara Asbrand, Christian Spieß

Julia Demmer

Jutta Wiesemann, Jochen Lange
Räume, Dinge und schulisches Wissen. Materials

„Verdammtes Ding, dich könn’ wir dann erforschen!“ Perspektiven auf kindliche Auseinandersetzungen mit den Dingen in institutionellen Kontexten

Lernen mit Dingen - Prozesse zirkulierender Referenz im Unterricht

Selbstpräsentationen von Zeitzeug_innen.

Fallrekonstruktion Schule

„Education in a Box“ Die Herstellung schulischer

80

Artefakte in der Lehr-Lernmittelindustrie

\section{Allgemeiner Teil}

Wiebke Bobeth-

Neumann

Anne Niessen
Angebote zur Qualifizierung für den

Schulleitungsberuf: Deutungsmuster von Lehrkräften auf dem Weg ins Schulleitungsamt

Musikpädagogische Perspektiven auf Heterogenität 106 und die Ambivalenz der Anerkennung. Ergebnisse einer qualitativen Interviewstudie mit Lehrenden des Programms ,Jedem Kind ein Instrument“ 
Sammy Wafi, Markus Wirtz

\section{Rezensionen}

Svenja Strauß

Till-Sebastian Idel
Visualisierungskompetenz in Deutsch und Mathematik aus Sicht von Expertinnen und Experten der Lehr-/Lernforschung und Fachdidaktik

Kade, Jochen/Nolda, Sigrid/Dinkelaker, Jörg/

Herrle, Matthias (Hrsg.) (2014): Videographische

Kursforschung. Empirie des Lehrens und Lernens

Erwachsener. Stuttgart: Kohlhammer Verlag

Zeitler, Sigrid/Heller, Nina/Asbrand, Barbara (2012): Bildungsstandards in der Schule. Eine rekonstruktive Studie zur Implementation der Bildungsstandards. Münster/New York/München/Berlin: Waxmann. ISBN 978-3-8309-2664-1. 


\section{Visualisierungskompetenz in Deutsch und Mathematik aus Sicht von Expertinnen und Experten der Lehr-/Lernforschung und Fachdidaktik}

\section{Zusammenfassung}

Visualisierungen sind von zentraler Bedeutung im Schul- und Lernalltag. Obwohl Visualisierungen zunehmend in der bildungswissenschaftlichen Forschung berücksichtigt werden, existiert bisher keine pädagogisch-psychologische und fachdidaktische Modellbasis, welche die erforderlichen Kompetenzen zur lernförderlichen Verarbeitung in disziplinübergreifender Anwendungsperspektive beinhaltet. Visualisierungskompetenz wird in diesem Beitrag als Fähigkeit zum Umgang mit externalen, statischen Visualisierungen verstanden, so dass diese dem Auf- und Ausbau adäquater Wissensstrukturen dienen. Der Beitrag strebt auf Basis einer qualitativen Befragung von Expertinnen und Experten sowie deren inhaltsanalytischer Auswertung die Entwicklung eines Strukturmodells von Visualisierungskompetenz im schulischen Kontext an, das (1) den internationalen erziehungswissenschaftlichen Diskurs zum Thema Visualisierungskompetenz integriert und (2) die Sichtweisen von Expertinnen und Experten mit Fokus auf Deutsch- und Mathematikunterricht berücksichtigt. Das resultierende Strukturmodell trägt zur fächerübergreifenden, konzeptuellen Klärung von Visualisierungskompetenz bei und liefert die Basis für ein empirisch fundiertes Diagnoseinstrumentarium.

Schlagwörter: Visualisierungskompetenz, Strukturmodellierung, Literaturanalyse, Expertenbefragung

\section{Competence in visualization for German and Mathematics from the viewpoint of teaching and learning as well as teaching methodology experts}

Visual representations enjoy central importance in everyday life and education. Despite their increasing consideration in education research, a pedagogical-psychological and subject-oriented modelling basis for the required competencies of visual literacy is lacking. This modelling basis would focus on an application-oriented, cross-disciplinary perspective and contain the competencies required for processing external, static visual representations in order to support learning and to develop and expand adequate structures of knowledge. Based on interviews with experts and their contentanalytical evaluation, this article aims at developing a structural model of visual literacy in schooling that (1) integrates the international educational discourse and (2) takes into account expert opinions with a focus on education in German and Mathematics. The resulting structural model contributes to the interdisciplinary, conceptual clarification of visual literacy and provides the basis for an empirically founded diagnostic tool.

Keywords: Visual Literacy, Structural Modeling, Literature Research, Expert Consultation

\section{Einleitung}

\subsection{Forschungsanlass und Ziele}

Die Verwendung, Interpretation und Konstruktion von visuell abbildbaren Informationen ist bei schulischen Lernprozessen von hoher Bedeutung (Schnotz 2010; Mayer 2005; Weidenmann 1994). Aufgrund verbesserter technischer Möglichkeiten bei Erstel- 
lung und Druck von Lernmaterialien sowie der Verwendung neuer Medien bestehen heute vielfältige Möglichkeiten zum Einsatz von Visualisierungen. Dadurch erhalten Visualisierungen eine Schlüsselfunktion für den Wissenserwerb (Kaput 1987; OECD 2004).

Visualisierungen in Lehr- und Lernkontexten sind einerseits Bestandteil der produktorientierten Schulbuchforschung (Doll \& Rehfinger 2012), welche Schulbücher als Unterrichtsmedien und Mittel der visuellen Kommunikation untersucht (Weinbrenner 1995). Visualisierungen erfüllen dabei verschiedene pädagogische Funktionen: sie dienen der Veranschaulichung, sind Teil von Aufgabenstellungen, enthalten Zusatzinformationen, dienen der Aktivierung vorherigen Wissens und der Einführung neuen Wissens, erlauben Vergleiche und die Demonstrierung von Verhältnissen, erklären Prozesse und Probleme und vermitteln Werte - jeweils mit variabler Komplexität und Verständlichkeit (Fischer-Dardai \& Kojanitz 2010). Andererseits sind Visualisierungen heute häufig in einen Kontext vielfältiger, multimedialer Lernumgebungen eingebettet und versprechen einen verbesserten Wissenserwerb aufgrund auditiver, visueller und audiovisuelle Wahrnehmungen. Um für Lernprozesse effektiv zu sein, müssen verschiedene Aspekte berücksichtigt werden. Nach den Multimediaprinzipien nach Mayer (2005) gehören dazu beispielsweise das Kontiguitätsprinzip (räumliche und zeitliche Nähe) und das Modalitätsprinzip (gesprochene statt geschriebene Erläuterungen zu Grafiken versprechen höheren Lernerfolg).

Neben Anforderungen an die Gestaltung der Visualisierungen selbst bedarf es zielgerichteter Untersuchungen zu den erforderlichen und vorhandenen Fähigkeiten auf Seiten der Schülerinnen und Schüler bei der Arbeit mit Visualisierungen, welche im Folgenden als Visualisierungskompetenz bezeichnet werden.

Während Kompetenzen im Umgang mit Texten (,literacy“, Textverständnis) und die dazu erforderlichen mentalen Modelle Gegenstand vielfältiger Forschungstätigkeiten sind (Krapp \& Weidenmann 2006), ist der Kenntnisstand zum Umgang mit Visualisierungen nicht gleichermaßen ausgeprägt (Ploetzner et al. 2013). Inzwischen liegt hinreichende empirische Evidenz vor, dass Visualisierungskompetenz wegen grundlegender Unterschiede in der Verarbeitung und in den mentalen Systemen bei Text- und Bilddarstellungen in Abgrenzung zu Literacy aufgefasst werden muss (Schnotz \& Bannert 2003; Schnotz 2010; Hochpöchler et al. 2013). Beispielsweise ist bei Visualisierungen im Gegensatz zu Texten für die semantische Verarbeitung im Allgemeinen keine bestimmte Bearbeitungsreihenfolge vorgesehen (Schnotz 2010) und es werden gemäß der Theorie der dualen Kodierung nach Paivio (1990) unterschiedliche Verarbeitungskanäle angesprochen.

In diesem Beitrag wird Visualisierungskompetenz als Fähigkeit zum - auch produktiven - Umgang mit Visualisierungen verstanden, so dass diese für Lern- und Aufgabenprozesse sinnvoll eingesetzt werden können und dem Aufbau adäquater Wissensstrukturen dienen. Das mit Visualisierungskompetenz eng verwandte Konzept der Visual Literacy hat im anglo-amerikanischen Raum eine längere, multidisziplinäre Forschungstradition, wobei besonders das Journal of Visual Literacy der International Visual Literacy Association (IVLA) hervorzuheben ist. Der Fokus liegt darin jedoch selten auf dem Schulkontext. Wie von Verschaffel et al. (2009) hervorgehoben existiert eine Forschungslücke in der Frage, wie im schulischen Lernkontext die erforderlichen Kom- 
petenzen für den Gebrauch flexibler Strategien auch bei (visuellen) Repräsentationen diagnostiziert und langfristig gefördert werden können.

Zum Schließen dieser Lücke werden im Folgenden die Extrahierung und Modellierung der Kompetenzen für den Umgang mit einfachen und multiplen externalen Visualisierungen zur Unterstützung schulischer Lernprozesse fokussiert. Die übergreifende Forschungsfrage lautet: Welche Kompetenzstruktur lässt sich für den Umgang mit Visualisierungen in den Fächern Deutsch und Mathematik in der Sekundarstufe I (mit Fokus auf der Orientierungsstufe) aus theoretischen und empirischen Erkenntnissen identifizieren?

Ziel ist die Entwicklung eines validen Kompetenzstrukturmodells (Klieme et al. 2007; Leuders 2014), d. h. die Identifizierung der Kompetenzdimensionen auf Seiten der Lernenden, welche bei externalen Repräsentationen wichtig sind, um fachliche Inhalte und Konzepte verstehen und anwenden zu können. Im Mittelpunkt des Interesses stehen bereichsübergreifende sowie fachspezifische Kompetenzmerkmale. Der Fokus liegt dabei auf den kognitiven Fähigkeiten der Lernenden, nicht auf dem eigentlichen Umgang oder den angewendeten Strategien.

Die Betrachtung beschränkt sich auf statische Visualisierungen (im Gegensatz etwa $\mathrm{zu}$ auditiven begleiteten und bewegten Materialien wie Filmen oder Simulationen), da diese (1) in Schulbüchern die vorherrschende Form von Visualisierungen in der Schuldidaktik darstellen und (2) eine Fokussierung des Visualisierungsaspekts ermöglichen, so dass dieser unabhängig von seiner Integration in komplexen, multimodalen Verarbeitungs- oder Handlungsregulationsmodellen behandelt werden kann (Calvert et al. 2004; Anderson 2010). Die Fragestellung wird mit Fokus auf Lernende der frühen Sekundarstufe 1 bearbeitet, da in diesem Alter typischerweise grundlegende kognitive Entwicklungsstufen erreicht sind, die für die Verarbeitung vieler Visualisierungen wichtig sind (insbes. das Stadium der formalen Operationen nach Piaget, Flammer 2009). Die Fächer Deutsch und Mathematik eignen sich fachlich für diese Thematik, da beide stark auf Visualisierungen angewiesen sind um abstraktes Wissen darzustellen und in beiden basale Kompetenzen auch für andere Unterrichtsfächer vermittelt werden.

Eine fundierte und empirisch abgesicherte Identifizierung von Visualisierungskompetenz und ihrer Dimensionen (Facetten) ist eine essentielle Voraussetzung für die Entwicklung eines validen Erfassungs- und Diagnoseinstrumentariums. Damit kann das Ziel verfolgt werden, Visualisierungskompetenz des Einzelnen in Wechselwirkung zum eigenen Wissenserwerb und Lernzuwachs zu setzen. Gelingt es, fachspezifische und fächerübergreifende Wissenszuwächse in Zusammenhang zu den identifizierten Ausprägungen von Kompetenzfacetten zu setzen, so ermöglichen die gewonnenen Ergebnisse Einsichten darin, welche Strategien für den Umgang mit Visualisierungen langfristig gefördert werden sollten und wie Lernumfelder gezielt für produktivere Lernprozesse gestaltet werden können. Die vorliegende Arbeit ist als grundlegender und zugleich notwendiger Schritt für diesen Prozess zu werten.

\subsection{Forschungsstand zu Visualisierungskompetenz}

Der vorliegende Beitrag fokussiert die Frage der Struktur von Visualisierungskompetenz. Obwohl die Modellierung von Kompetenzen in der Literatur oft mit einem Fokus 
auf psychometrischer Messung dargestellt und diskutiert wird, muss zur Sicherstellung der Validität eine fundierte qualitative Klärung des zugrundliegenden Konstrukts erfolgen. Nur wenn die Kernmerkmale und -prozesse der kognitiven Verarbeitungsprozesse identifiziert werden, kann eine angemessene Entwicklung von Aufgaben erfolgen. Diese müssen so formuliert werden, dass die für den Kompetenzbereich kritischen kognitiven Prozesse ausgelöst werden und lösungsdeterminierend sind. Qualitative kognitive Interviews, z. B. Comprehension Probing und Think-Aloud-Technik (Charters 2003), sowie Experteninterviews, die der konsensuellen und kommunikativen Validierung dienen, stellen einen notwendigen Entwicklungsschritt für Kompetenzstruktur- und Kompetenzniveaumodelle dar (Klieme et al. 2007).

Kompetenzen sind allgemein Fähigkeiten, die es Individuen ermöglichen, mit Anforderungen in spezifischen Situationen umzugehen (Klieme et al. 2007). Sie werden von generalisierten, kontextunabhängigen kognitiven Leistungskonstrukten (wie Intelligenz) abgegrenzt (Prenzel et al. 2007). Dieser Beitrag betrachtet Kompetenzen als verfügbare Leistungsdispositionen (über welche kognitiven Kompetenzen verfügt ein Individuum zu einem bestimmten Zeitpunkt?) und zielt auf die Binnenstruktur der Kompetenzbereiche ab (wie lassen sich Teilkompetenzen oder Kompetenzfacetten sinnvoll repräsentieren und differenziert ausgestalten?).

Visualisierungen - auch als visuelle Darstellungen oder Repräsentationen bezeichnet - gehören zu den informierenden Bildern nach Weidenmann (1994a) und dienen der Informationsvermittlung. Im Gegensatz zu künstlerischen und unterhaltenden Bildern sind für informierende Bilder Klarheit und angemessener Informationsgehalt für die Lernenden zentrale Zielkriterien. Im Allgemeinen sind diese Darstellungen eindeutig in Bezug auf eine intendierte, korrekte Rezeptionsweise. Informierende Bilder lassen sich in ikonische (realistische, bildliche) Darstellungen (wie Fotos, Skizzen, Bildergeschichten, Piktogramme) und symbolische (logische, analytische, beschreibende) Darstellungen (wie semantische Netze, Baumstrukturen, Flussdiagramme, Kurven) unterscheiden (Schnotz 2010; Weidenmann 1991). Sofern kein inhaltlich notwendiger Bezug von der Darstellung zum Lerninhalt oder der Aufgabenstellung festzustellen ist, werden dekorative Bilder oder Illustrationen in dieser Arbeit nicht einbezogen.

Visualisierungskompetenz hat primär im Rahmen von Visual Literacy seit der Verwendung von Debes im Jahr 1969 Verbreitung gefunden. In seiner ursprünglichen, vorläufigen Eingrenzung bezeichnet Visual Literacy eine Gruppe von Kompetenzen, die es einer Person ermöglicht, sichtbare Handlungen, Gegenstände und Symbole zu unterscheiden und interpretieren, um mit anderen kommunizieren sowie Werken Verständnis und Wertschätzung entgegenbringen zu können (Debes 1970). Wileman (1993) spricht bei Visual Literacy von der Fähigkeit "to ,read, interpret, and understand information presented in pictorial or graphic images" (114). Er sieht Visual Literacy als rezeptive Gegenkomponente zum produktiven Visuellen Denken, d. h. der Fähigkeit, Informationen jeglicher Art in Bilder, Grafiken oder andere Formen zu übertragen. Paquin (1999) kombiniert die Perspektiven mehrerer Autoren mit Visuellem Denken, Visuellem Lernen und Visuellem Kommunizieren nach Seels (1994) und ergänzt sie durch eine Taxonomie der Ergebnisse von Visual Literacy auf verschiedenen Schwierigkeitsstufen. Bis heute konnte jedoch keine einheitliche Definition von Visual Literacy ermittelt werden (Avgerinou \& Ericson 1997; Avgerinou 2001; Brill et al. 2007). Dies ist in erster Linie der 
Vielfalt an Disziplinen geschuldet, die diesen Begriff verwenden; dazu gehören Kunst ebenso wie z. B. Naturwissenschaft, Wirtschaft, Linguistik und Psychologie.

Dennoch herrscht in der Literatur weitgehend Einigkeit, dass Visual Literacy lehrund lernbare kognitive, teilweise auch affektive, Fähigkeiten umfasst, welche Aspekte (a) des Lesens, Dekodierens und Interpretierens von visuellen Darstellungen, (b) des Schreibens, Kodierens und Erschaffens von visuellen Darstellungen sowie (c) die Fähigkeit des visuellen Denkens beinhaltet (Avgerinou 2001; Avgerinou \& Pettersson 2011). Zahlreiche Arbeiten gehen davon aus, dass Visual Literacy aus mehreren Facetten oder Dimensionen besteht (,group of abilities“, Avgerinou 2001), auch wenn dies nicht immer expliziert und von einer ,ability of visual literacy“ gesprochen wird. Unklarheit herrscht nach wie vor darüber, wie sich die Facetten oder Dimensionen definieren, abgrenzen und operationalisieren lassen.

Untersuchungen im Schulkontext zu schulischen Lernprozessen mit Visualisierungen legen den Schwerpunkt häufig auf multiple Repräsentationen (de Jong et al. 1998; Ainsworth 1999, 2006; van der Meij 2007), i. d. R. mit Fokus auf Mathematik und Naturwissenschaft. Während de Jong et al. die Dimensionen der Visualisierungen selbst ins Zentrum rücken (Perspektive, Genauigkeit, Ausführungsart, Ausprägung, Schwierigkeitsgrad), beschreiben Ainsworth und van der Meij Funktionen multipler Visualisierungen (Informationsergänzung, Einschränkung der Interpretationsmöglichkeiten, tiefere Verständniskonstruktion) und nötige Kompetenzen auf Seiten der Lernenden, jedoch ohne theoriebasierte Herleitung oder empirische Untersuchung. Demnach müssen Lernende das Darstellungsformat kennen, die Beziehung zwischen der Repräsentation und dem Fachbereich verstehen, geeignete Repräsentationen auswählen sowie selbst erstellen können (Ainsworth 2006). Laut Ainsworth (2006) ist es unwahrscheinlich, dass eine einfache Beziehung zwischen gelingender Rezeption und der Produktion von Visualisierungen besteht.

Für die Aneignung von Wissen ist es nicht immer hilfreich, Texte mit Visualisierungen auszustatten, da sich unvorteilhafte Darstellungen auch nachteilig auf die Herausbildung passender mentaler Modelle auswirken können (Schnotz \& Bannert 2003; Watkins et al. 2004). Jedoch sind wirkungsvolle kognitive Techniken und Strategien für die Text-Bild-Integration in Abgrenzung zum Umgang mit Texten notwendig, z. B. zum eigenständigen Verbinden und Vergleichen von verbalen und visuellen Einheiten, Visualisieren von Text und Verbalisieren von Bildern. Ploetzner et al. (2013) diagnostizieren hier ein Forschungsdefizit.

Stokes (2002) zieht bei der literaturbasierten Analyse von empirischen Forschungsarbeiten aus dem Schul- und Lehrbereich das Fazit, dass visuelle Elemente beim Lehren und Lernen im Allgemeinen positive Ergebnisse erzielen. Die einbezogenen Arbeiten lassen vermuten, dass die Resultate vom Vorwissen der Lernenden (ChanLin 1998, McKay 1999), jedoch nicht unbedingt von Visualisierungspräferenzen (Smaldino \& Russell 2005) abhängen.

Die von Presmeg (2006) formulierten Forschungsdesiderate zu Visualisierungskompetenzen für den Mathematikunterricht sind insofern gültig, als dass bis heute kein Diagnoseinstrumentarium für den Schulunterricht existiert und keine Arbeiten bekannt sind, die Visualisierungskompetenz bei Lernenden in den Fächern Deutsch und Mathematik kombiniert betrachten. 


\section{Modellierung der Struktur von Visualisierungskompetenz}

Modellentwicklung. Als Ausgangspunkt zur Konstruktion des Strukturmodells diente eine literaturbasierte Identifikation relevanter Kompetenzfacetten. Dafür wurde zunächst umfassende Literatur zur Thematik gesammelt (u. a. Artikel im Journal of Visual Literacy 1990-2013; Ainsworth 1999, 2006; Avgerinou 2001, 2007; Debes 1970; Dondis 1973; Fransecky und Debes 1972; Paquin 1999; Seels 1994; van der Meij 2007; Wileman 1993; s. Tab. 2). Die Arbeiten wurden nach relevanten Aspekten gesammelt und geordnet, konsensuell in der Forschergruppe nach ihrer Bedeutung gruppiert und eine erste Kategorisierung vorgenommen. Durch eine Gegenüberstellung einzelner Arbeiten konnten die Kernelemente synoptisch verglichen werden. Es stellte sich heraus, dass Begrifflichkeiten verschiedener Autoren semantische Übereinstimmungen aufweisen, wodurch sie zusammengefasst oder generalisiert werden können. Dies war z. B. der Fall bei ,form“ und ,format“ (Ainsworth 2006), ,syntax“ (van der Meij 2007), „knowledge of visual vocabulary“ und „knowledge of visual conventions“ (Avgerinou 2001), welche bereits bei Debes (1970) als ,know the grammar and syntaxes of visual language“ (13) und Fransecky (1970) als „knowledge“ auftreten. Sie wurden als „Kenntnis des Darstellungsformats“ (nach der Expertenbefragung bezeichnet als „Erkennen der Darstellung") generalisiert. Alle weiteren Zusammenfassungen, Generalisierungen und Konkretisierungen sind in Tab. 2 dargestellt.

Im Verlauf der literaturbasierten Informationsintegration gab es drei zentrale Kriterien:

- Visuelle Unterscheidung (von Farben, Formen, Helligkeiten, „skills“ nach Debes 1970, ,visual discrimination“ nach Avgerinou 2001) wurde als grundlegende, physische Voraussetzung von Visualisierungskompetenz angesehen (,Sehen können“). Dies impliziert, dass die Kenntnis des Darstellungsformats auch das Erkennen dieses Formats anhand einer gegebenen Visualisierung möglich macht und es nicht am Nicht-Erkennen von Formen und Farben scheitert (wie Rot-Grün-Schwäche).

- Es wurden nur für den gegebenen Kontext maßgebliche Aspekte einbezogen. Insbesondere wurden rein künstlerische Aspekte nicht gesondert betrachtet (wenig Relevanz für Deutsch und Mathematik).

- Es wurden nur externale oder external erkennbare Aspekte integriert. Rein mentale Prozesse wie Visualisieren und Visuelles Denken (Avgerinou 2001, ,visualization“, „,visual thinking“) wurden im Strukturmodell nicht abgebildet; sie haben jedoch Einfluss auf enthaltene Kompetenzfacetten wie „Verstehen“. Ebenso wird der Aspekt des Visuellen Erinnerns als primär mentaler Prozess im Strukturmodell nicht expliziert. Visuelles Erinnern als bildliches Erinnerungsvermögen oder Abrufenkönnen bildlicher Informationen nimmt bereits im Visual Literacy Index nach Avgerinou eine Sonderstellung ein. Für die Tatsache, dass es in ihrem (nicht schulspezifischen) Test nicht empirisch bedeutsam unterschieden werden konnte, gibt Avgerinou zwei Erklärungsmöglichkeiten: Visuelles Erinnern sei entweder nicht essentiell für das Erfassen der Ausprägung von Visual Literacy oder es sei in jeder einzelnen Fähigkeit subsumierend eingebettet (Avgerinou 2001: 478). 
Diesen Regeln entsprechend stellten sich z. B. von den fünf Einzelkomponenten von Brill et al. (2007) nur zwei, nämlich Erzeugen und Verstehen, als bedeutsam für diese Arbeit heraus, während die anderen entweder als Voraussetzung gelten (Unterscheiden), im konkreten Zusammenhang wenig Relevanz haben (Wertschätzen) oder sich auf rein mentale Vorstellungen (,conjure“) beziehen. Sofern Wertschätzung sich auf motivationale Aspekte auswirkt, ist jedoch von indirektem Einfluss auszugehen, der dann wiederum auf die Performanz Einfluss haben kann.

Die so in mehreren Stufen ermittelten Facetten wurden anhand zufällig ausgewählter Visualisierungsbeispiele aus mehreren Schulbüchern (für Deutsch und Mathematik) exemplarisch geprüft und gegebenenfalls modifiziert oder korrigiert. Sie konnten vier Kategorien zugeteilt werden: „Kenntnis des Darstellungsformats“, „Verstehen der Darstellung“, „Assoziieren von Darstellungen“ und „Generieren von Darstellungen“ (Arbeitsbegriffe; nach der Expertenbefragung teilweise durch die in Tab. 2 verwendeten Bezeichnungen substituiert). Anhand der ermittelten Facetten, ihrer Kategorisierung und Strukturierung wurde eine tabellarische Übersicht als Grundlage entwickelt und eine erste Version des Strukturmodells daraus abgeleitet.

Verwandte Konstrukte. In Zusammenhang mit Visualisierungskompetenz stehen weitere Konstrukte und Konzepte. Dazu gehören Räumliches Vorstellungsvermögen und Abstraktes Denken ebenso wie fachliches Vorwissen und Intelligenz. Die Art des nomologischen Netzes von Visualisierungskompetenz ist eine in der Literatur unbeantwortete Fragestellung und dadurch Teil der weiteren Forschungsarbeit.

\section{Expertenbefragung}

Zur weiteren empirischen Entwicklung des durch die literaturbasierte Informationsintegration hergeleiteten Strukturmodells wurde eine leitfadenbasierte Expertenbefragung anhand fokussierter Interviews durchgeführt. Durch die Auswahl der qualitativen Inhaltsanalyse als deduktiv-induktives Verfahren werden explizit verbalisierte Äußerungen der Experten strukturiert fokussiert.

\subsection{Expertinnen- und Expertenauswahl und Befragungszeitpunkt}

Die Befragung fand in Form von Einzelinterviews mit 14 Expertinnen und Experten in Baden-Württemberg und Nordrhein-Westfalen zwischen November 2013 und Januar 2014 statt. Die Interviews dauerten zwischen 25 und 70 Minuten (Durchschnittsdauer von etwa 45 Minuten).

Es wurden einerseits international anerkannte Expertinnen und Experten im Bereich der Lehr-/Lernforschung und fachdidaktisch orientierten Visualisierungsforschung befragt, die in angesehenen nationalen und internationalen Fachzeitschriften publizieren. Zusätzlich wurden Lehrpersonen einbezogen, die sich durch ihr fachdidaktisch-spezifisches Erfahrungswissen auszeichnen und deren mehrjährige Berufserfahrung es erlaubt, Kompetenzen und Schwierigkeiten der Schülerinnen und Schüler beurteilen zu können. Die Einbeziehung von Forschenden sowie Lehrpersonen sollte einen breiten Einblick in die Thematik sowie die Berücksichtigung verschiedener Perspektiven ermöglichen. 
Von den Expertinnen und Experten waren sechs männlich und acht weiblich; sieben als Professor/in, drei als wissenschaftliche(r) Mitarbeiter/in und vier als Lehrpersonen tätig. Fünf hatten den primären Fachbereich Deutschdidaktik, vier Mathematikdidaktik und fünf Medienpädagogik bzw. Psychologie.

Die Interviews wurden akustisch aufgezeichnet und anschließend transkribiert.

\subsection{Methodischer Ansatz und Vorgehen}

Methodischer Ansatz. Die Expertinnen und Experten wurden informiert, dass es in der Befragung um Fähigkeiten zum Einsatz von Visualisierungen für Lernprozesse auf Seiten von Schülerinnen und Schülern geht. Es wurde keine Beschränkung auf die Sekundarstufe I vorgenommen, auch wenn die präsentierten Visualisierungsbeispiele aus diesem Bereich gewählt wurden.

Bei der Befragung wurde auf eine maximale Offenheit geachtet (Bortz und Döring 2006; Reinders 2005). Es wurden Impulsfragen und unterstützende Materialien eingesetzt. Das Strukturmodell wurde den Expertinnen und Experten dabei zu keiner Zeit präsentiert. Stattdessen wurden die Befragten mit Hilfe der Strukturlegetechnik (Wahl 2006) mit einzelnen Begrifflichkeiten, Facettenvorschlägen und Konkretisierungen aus dem Strukturmodell konfrontiert, ohne Vorgaben oder Ideen zu ihrer Strukturierung zu machen. Die Expertinnen und Experten wurden ermuntert, die Begriffe zu verändern und sie als unverbindliche Vorschläge zu betrachten. Die vorgegebenen Kompetenzfacetten waren somit als Anstoß zum eigenen Denken und nicht als Vorgabe zu verstehen.

Vorgehen. Es wurde ein dreistufiger Befragungsansatz gewählt. Der erste phänomenologische Befragungsteil zu Erfahrungswerten mit Visualisierungen und ihrem schulischen Einsatz (sofern vorhanden) diente primär dem mentalen Wachrufen von Visualisierungsbeispielen und der für die zwei folgenden Teile der Befragung notwendigen Einstimmung auf die Thematik.

Im zweiten Teil wurden die modellierten Kompetenzfacetten unter Verwendung der Think-Aloud-Methode (Ericsson \& Simon 1980; Häder 2010) erfasst und vervollständigt. Dafür wurden die Expertinnen und Experten mit einer Auswahl an Visualisierungen aus Schulbüchern der Sekundarstufe I konfrontiert und aufgefordert, laut zu äußern, welche Fähigkeiten Lernende bei der Arbeit mit den Visualisierungen benötigen. Die dafür verwendeten Visualisierungen (je sechs für Deutsch und Mathematik) wurden mit Aufgabenstellungen vorgelegt. Kriterium für die Auswahl der Visualisierungen war die inhaltliche Relevanz für das Einführen neuer Sachinhalte oder für Aufgabenstellungen. Jede/r Expertin/e erhielt zwei bis drei Aufgaben zur Bearbeitung und jede Visualisierung wurde von zwei bis vier Expertinnen und Experten bearbeitet, wobei die Visualisierungen je nach Fachgebiet der/s Expertin/en zugeteilt wurden. Dieses Vorgehen anhand der Think-Aloud-Methode erlaubt die Thematisierung und Erfassung auch latenter Kompetenzen und Fertigkeiten, die auf direkte Fragen übersehen oder nicht unmittelbar verbalisiert werden. Dieser Teil diente zur Vervollständigung des Strukturmodells.

Im dritten Teil ging es um die Strukturierung, Konkretisierung, Beurteilung und Bewertung der modellierten Kompetenzfacetten unter Verwendung der Think-AloudMethode und Strukturlegetechnik. Beispielhafte Veranschaulichungen von vier Kompetenzfacetten anhand fachspezifischer Visualisierungen gaben den Impuls. Die Erläute- 
rung beschränkte sich auf die Ebene der Kompetenzfacetten; Konkretisierungen wurden nicht erklärt. Zudem wurden Erklärungskärtchen zu den einzelnen Kompetenzfacetten vor den Augen der Expertinnen und Experten gemischt, um keine Reihenfolge oder Ordnung zu suggerieren. Anschließend wurden die Expertinnen und Experten aufgefordert, aus den gegebenen Kärtchen mit Kompetenzen und Konkretisierungen sowie aus weiteren selbst hinzuzufügenden Facetten und Konkretisierungen eine Struktur zu legen. Dafür stand Material (Plakat, Kärtchen, Stifte, etc.) zur Verfügung, einschließlich leerer Kärtchen zum Vervollständigen und Erweitern. Während des Strukturlegens wurden die Expertinnen und Experten zum Äußern ihrer Gedanken ermuntert.

Zuletzt wurden kleine Nummernkärtchen ausgegeben und die Expertinnen und Experten gebeten, sich auf eine Ordnung der Kompetenzfacetten festzulegen, sofern dies möglich war und als sinnvoll erachtet wurde. Auch dies geschah kombiniert mit der Think-Aloud-Methode. Das Endresultat der strukturlegebasierten Modellierung wurde fotografisch festgehalten und in die Auswertung einbezogen.

\subsection{Auswertung der Expertenbefragung}

Die Transkriptionen der teilstrukturierten Interviews erzeugten umfassendes empirisches Material (über 160 Seiten), welches mittels einer qualitativen Inhaltsanalyse (Mayring 2010; Kruse 2014) ausgewertet wurde.

Abbildung 1: Methodisches Vorgehen zur Auswertung der Expertenbefragung

Transkriptionen

Inhaltsanalyse nach Mayring

Reduzierte und zusammengefasste

Transkriptionen

Inhaltl./typisierende Strukturierung

49 Einzelaspekte

Zweistufiges Komprimieren

18 mögliche Änderungspunkte

Plausibilitätsprüfung \& Sortieren

Liste mit Änderungen

Anpassung Modell \& Definitionen

Strukturmodell (Abb. 2) 
Die Auswertung des zweiten und dritten Teils der Expertenbefragung war mehrstufig (Ablauf s. Abb. 1). Die Transkriptionen wurden einer zusammenfassenden und explizierenden Inhaltsanalyse unterzogen. Durch eine strukturierende Inhaltsanalyse wurden von den Expertinnen und Experten genannte Facetten und Aspekte extrahiert. Eine inhaltliche und typisierende Strukturierung erlaubte die Sammlung ähnlicher Punkte und die Identifizierung häufig genannter Aspekte. Die zunächst 49 Änderungs- und Konkretisierungspunkte wurden durch Bereinigen von Mehrfachnennungen und Generalisieren in einem zweistufigen Vorgehen auf insgesamt 18 Punkte reduziert, welche anschließend nach Plausibilität sortiert und zur Vermeidung von Redundanzen aggregiert wurden.

Für die 18 extrahierten Punkte der Expertinnen und Experten wurde individuell entschieden, ob die Aspekte (1) im Strukturmodell Anpassungen erfordern, (2) in den Definitionen und Erläuterungen Änderungen implizieren, (3) Relevanz für eine mögliche Operationalisierung besitzen oder (4) nicht weiter einbezogen werden sollten (Mehrfachzuweisungen waren möglich). Die Punkte lassen sich in Kategorien einteilen und betreffen (A) begriffliche Anpassungen, (B) strukturelle Anpassungen, (C) neue Kompetenzfacetten oder Konkretisierungen, (D) Anpassungen in den Definitionen und Erläuterungen der Kompetenzfacetten sowie (E) keine Aufnahme ins Strukturmodell. Tab. 1 im Anhang liefert eine Übersicht über die Anpassungs- und Konkretisierungsaspekte und den gewählten Umgang mit ihnen. Davon ausgehend wurden die Definitionen der Kompetenzfacetten überarbeitet und gegebenenfalls konkretisiert.

Ein ähnliches zweistufiges Vorgehen wurde auch separat für die Erfassung der Ordnungskriterien und -resultate realisiert.

\subsection{Resultate der Expertenbefragung}

Neben der Überarbeitung des Strukturmodells ergaben sich durch die Expertenbefragung folgende weiteren Ergebnisse:

1. Die Befragung legt nahe, dass es nur geringe Überschneidungen in den Darstellungsarten zwischen Deutsch und Mathematik gibt. Einzig Tabellen und Diagramme wurden beide in Deutsch sowie in Mathematik genannt und können diesbezüglich als fächerübergreifende Formen von Visualisierungen betrachtet werden. Andere Darstellungsarten sind fachspezifisch (wie Mindmaps, Zahlenstrahl, Schrägbilder) oder themenspezifisch (wie Baumstrukturen).

2. Ein möglicher Unterschied zwischen Deutsch und Mathematik besteht darin, dass Visualisierungen in Mathematik sowohl Lerngegenstand (Diagramme, Funktionsgraphen, etc.) als auch Lernhilfe (Kuchenmodell bei Brüchen) sind, während sie in Deutsch meistens als Lernhilfe auftreten.

3. Verstehen von Darstellungen steht gerade in Deutsch häufig mit Interpretation, Hinterfragen des Erkannten und kritischem Betrachten in Zusammenhang. Kritisches Betrachten (,critical viewing“ nach Avgerinou 2001) ist der Kompetenzfacette „Verstehen der Darstellung“ zuzurechnen, sofern es sich auf die dargestellten Inhalte bezieht. Geht es um subtile Intentionen - wenn z. B. Ziele im Werbeumfeld verfolgt oder Gender- bzw. Minderheitsstereotypen suggeriert werden - so ist dies vor allem für höhere Schulstufen und spätere Lebensphasen von Bedeutung. 
4. Das Verhältnis der Kompetenzfacetten untereinander lässt sich durch die Expertenbefragung nicht beantworten. Bei der Hierarchisierung nahm die Hälfte der Expertinnen und Experten eine Ordnung nach dem kognitiven Anspruchsniveau vor, die anderen sortierten aufgrund der kulturspezifischen Entwicklung beim Menschen (allgemein und beim Spracherwerb), anhand des Lernprozesses oder nach dem Grad der Abstraktion. Einzig „Erkennen der Darstellung“ wurde als grundlegende Kompetenzfacette, auf welche andere Kompetenzfacetten aufbauen, weithin konsistent formuliert.

\subsection{Kommunikative Validierung der Ergebnisse mit Expertinnen und Experten}

Zum Abschluss des empirischen Teils dieser Untersuchung wurde eine Diskussion in der Gesamtgruppe zur finalen Bewertung des entwickelten Strukturmodells durchgeführt. Dabei waren einige der bereits befragten sowie weitere Expertinnen und Experten anwesend, von denen einige in ein größeres, vom Wissenschaftsministerium des Landes gefördertes Forschungsprojekt zum Thema Visualisierungen eingebunden sind. In dieser Diskussionsphase wurde den Expertinnen und Experten das entwickelte Strukturmodell vorgelegt und intensiv diskutiert. Kleinere Konkretisierungen - vor allem wegen Missverständnissen aufgrund verschiedener fachlicher Hintergründe - wurden im Modell und der Beschreibung der Facetten angepasst. Es wurde ein allgemeiner Konsens zum Modell erreicht.

\section{Entwickeltes Strukturmodell von Visualisierungskompetenz}

Durch die literaturbasierte Informationsintegration, die exemplarische Prüfung anhand von Visualisierungen in Schulbüchern, individuelle Expertenbefragungen sowie die Diskussion in der Gesamtgruppe mit Visualisierungsexpertinnen und -experten konnte das in Abb. 2 dargestellte Strukturmodell von Visualisierungskompetenz erstellt werden. Visualisierungskompetenz wird demnach verstanden als ein Konstrukt bestehend aus vier Facetten, von denen sich drei einer rein rezeptiven Komponente und die vierte einer produktiven Komponente zuordnen lassen. Die Facetten werden in Tab. 2 aufgelistet und definiert sowie im Folgenden auch anhand einzelner Beispiele aus den Bildungsstandards der KMK (KMK 2004) erläutert. 
Abbildung 2: Strukturmodell von Visualisierungskompetenz

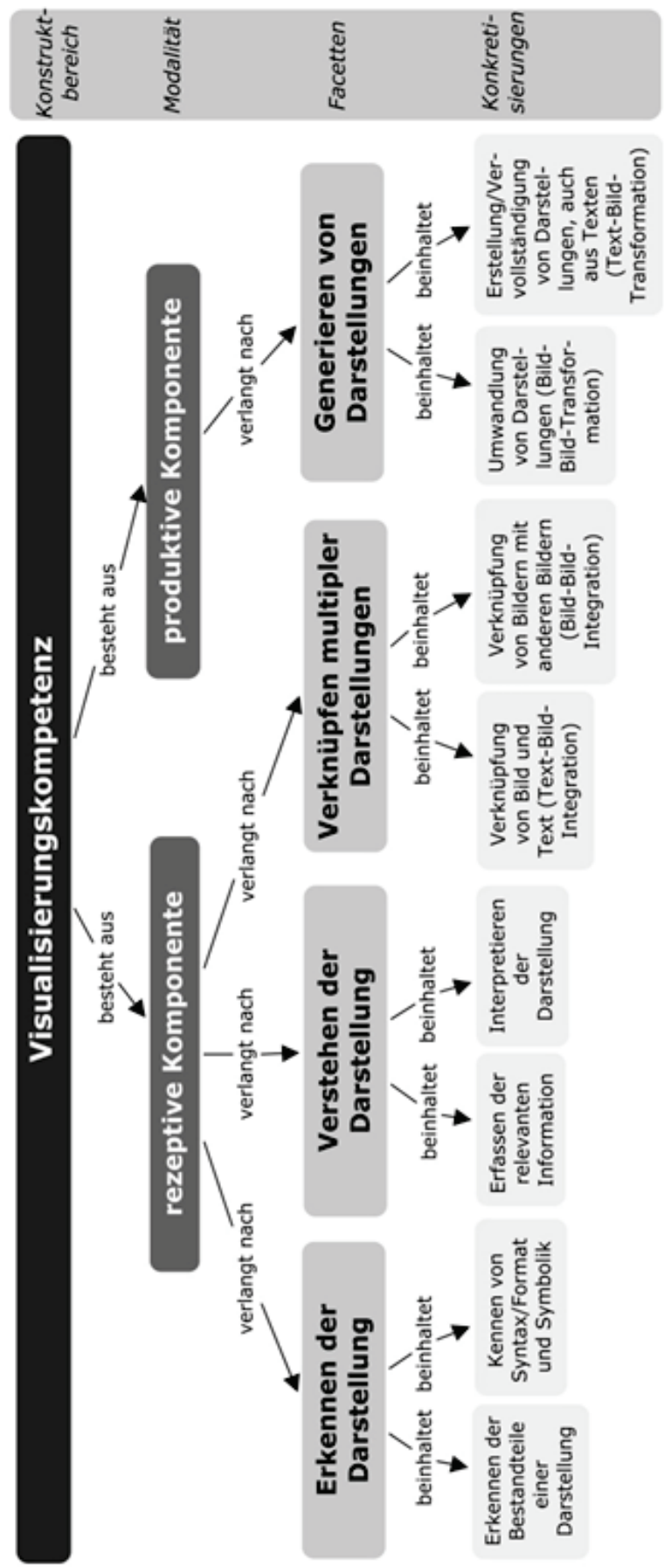


Tabelle 2: Definition der Facetten von Visualisierungskompetenz mit Konkretisierungsbeispielen

\begin{tabular}{|c|c|c|c|}
\hline $\begin{array}{l}\text { Kompetenz- } \\
\text { facette }\end{array}$ & Definition & Literaturbasis & $\begin{array}{l}\text { Konkretisierungsbeispiele } \\
\text { ( } M=\text { Mathematik, } \\
D=\text { Deutsch, } \\
\text { sonst fächerübergreifend) }\end{array}$ \\
\hline $\begin{array}{l}\text { Erkennen } \\
\text { der } \\
\text { Darstellung }\end{array}$ & $\begin{array}{l}\text { Erkennen der } \\
\text { abgebildeten } \\
\text { Bestandteile und } \\
\text { Kennen ihrer fach- } \\
\text { spezifischen Syntax } \\
\text { und Symbolik, ein- } \\
\text { schließlich Zeichen } \\
\text { und Symbolen }\end{array}$ & $\begin{array}{l}\text { „form/format“ (Ainsworth 2006: 4); } \\
\text { „syntax“ (van der Meij 2007: 17); } \\
\text { „knowledge of visual vocabulary“ } \\
\text { und „knowledge of visual conven- } \\
\text { tions“ (Avgerinou 2001: 414); „,know } \\
\text { the grammar and syntaxes of visual } \\
\text { language“ (Debes 1970: 13); } \\
\text { „knowledge“ (Fransecky 1970: 154) }\end{array}$ & $\begin{aligned} \text { - } & \text { Finden und Erkennen von } \\
& \text { Bildelementen } \\
\text { - } & \text { Erkennen eines Koor- } \\
& \text { dinatensystems oder } \\
& \text { Kreisdiagramms (M) } \\
\text { - } & \text { Erkennen von } \\
& \text { Einzelmimik/-gestik (D) }\end{aligned}$ \\
\hline $\begin{array}{l}\text { Verstehen } \\
\text { der } \\
\text { Darstellung }\end{array}$ & $\begin{array}{l}\text { Inhaltliches } \\
\text { Erfassen des } \\
\text { dargestellten } \\
\text { Sachverhalts } \\
\text { durch Einbeziehen } \\
\text { der relevanten } \\
\text { Information und } \\
\text { Interpretation ihrer } \\
\text { Bedeutung }\end{array}$ & $\begin{array}{l}\text { „comprehend“ (Brill et al. 2007: } \\
\text { 56); „comprehension: translation, } \\
\text { interpretation, extrapolation“ } \\
\text { (Fransecky 1970: 155); „interpretati- } \\
\text { on of representations“ und „domain“ } \\
\text { (Ainsworth 2006: 4f.); „,constructing } \\
\text { meaning“, „, (verbo-)visual rea- } \\
\text { soning“, „visual reconstruction“, } \\
\text { "critical viewing“ (Avgerinou 2001: } \\
\text { 414-416); „understanding which } \\
\text { parts of the domain are represented“ } \\
\text { (van der Meij 2007: 18); „interpret“ } \\
\text { and „understand“ (Wileman 1993: } \\
\text { 114); „[...] makes optimal use of } \\
\text { all available information“ (Legge } \\
\text { et al. 1989: 365); Unterscheidung } \\
\text { ikonischer und symbolischer Dar- } \\
\text { stellungen/,Graph-als-Bild“-Fehler } \\
\text { (Eichler \& Vogel 2013) }\end{array}$ & $\begin{array}{l}\text { - Verbinden mehrerer } \\
\text { Elemente einer Darstel- } \\
\text { lung zu einer gemein- } \\
\text { samen Bedeutung } \\
\text { - Unterscheiden ikonischer } \\
\text { und symbolischer Dar- } \\
\text { stellungen } \\
\text { - Verstehen der Datenver- } \\
\text { teilung in Grafiken (M) } \\
\text { - Antwort auf Verständnis- } \\
\text { fragen zu Bildern (D) }\end{array}$ \\
\hline $\begin{array}{l}\text { Verknüpfen } \\
\text { multipler } \\
\text { Dar- } \\
\text { stellungen }\end{array}$ & $\begin{array}{l}\text { Verbinden von } \\
\text { Visualisierungen } \\
\text { oder von Texten und } \\
\text { Visualisierungen, } \\
\text { Verstehen ihrer } \\
\text { Beziehung sowie } \\
\text { Erschließen des } \\
\text { gemeinsamen Be- } \\
\text { deutungsgehalts }\end{array}$ & $\begin{array}{l}\text { „translation“, „see the relation“ } \\
\text { (Ainsworth 1999: 132); „relating } \\
\text { representations“ (van der Meij } \\
\text { 2007: 17f.); „(sensitivity) to visual } \\
\text { association“" und „(sensitivity to) } \\
\text { verbo-visual association“ (Avgerinou } \\
\text { 2001: 415); „combine visuals and } \\
\text { verbals for intentional communica- } \\
\text { tion“ (Fransecky \& Debes 1972: 12); } \\
\text { "text-and-picture-comprehension“ } \\
\text { (Schnotz \& Bannert 2003: 141) }\end{array}$ & $\begin{array}{l}\text { - Text-Bild-Integration: } \\
\text { kohärentes Erfassen } \\
\text { von Darstellung(en) und } \\
\text { zugehörigem Text } \\
\text { - } \text { Bild-Bild-Integration: } \\
\text { Darstellungen aufstei- } \\
\text { gend sortieren (M), Bilder } \\
\text { einer Bildergeschichte in } \\
\text { eine logische Reihenfol- } \\
\text { ge bringen (D) }\end{array}$ \\
\hline $\begin{array}{l}\text { Generieren } \\
\text { von Dar- } \\
\text { stellungen }\end{array}$ & $\begin{array}{l}\text { Eigenständiges } \\
\text { Erstellen geeigneter } \\
\text { Visualisierungen } \\
\text { und Vervollständi- } \\
\text { gen bestehender } \\
\text { Visualisierungen } \\
\text { aufgrund gegebener } \\
\text { oder bekannter } \\
\text { Sachverhalte }\end{array}$ & $\begin{array}{l}\text { „compose“ (Debes 1970: 12f.), } \\
\text { „construct an appropriate repre- } \\
\text { sentation“(Ainsworth 2006: 5); } \\
\text { „visualization” und „visual thinking“ } \\
\text { (Avgerinou 2001: 414), „,create“ (Brill } \\
\text { et al. 2007: 55), „generate images for } \\
\text { communicating ideas and concepts“ } \\
\text { (Stokes 2002: 10); „drawing } \\
\text { strategy“ (van Meter 2001: 131) }\end{array}$ & $\begin{array}{l}\text { - Text-Bild-Transformation: } \\
\text { Erstellen von Mind-Maps } \\
\text { aufgrund von Texten oder } \\
\text { Vorwissen (D) } \\
\text { - Bild-Bild-Transformation: } \\
\text { Fahrtenschreiber in } \\
\text { Koordinatensystem } \\
\text { umwandeln (M) }\end{array}$ \\
\hline
\end{tabular}


1. „ErkennenderDarstellung“bezeichnetErkennenderineinerVisualisierungabgebildeten Bestandteile sowie Kennen ihrer fachspezifischen Syntax und Symbolik.

Dies beinhaltet Zeichen und Symbole, die als fächerübergreifende oder fachspezifische Konventionen verstanden werden (wie Winkelzeichen und Wurzelzeichen in Mathematik oder Sprechblasen und Abkürzungen für Wortarten in Deutsch). Dazu gehören beispielsweise das Finden und Benennen von Bildelementen als fächerübergreifende Komponente und - als fachspezifische Komponenten - das Ablesen von Einzelinformationen aus Grafiken für Mathematik sowie das Erkennen der Mimik und Gestik einzelner Personen in Visualisierungen für Deutsch. Erkennen gilt dabei gleichermaßen für ikonische wie symbolische Bildelemente (wie Koordinatensysteme, Säulen-/Kreisdiagramme, Mindmaps).

2. „Verstehen der Darstellung“ bezeichnet inhaltliches Begreifen des in einer einzelnen Visualisierung dargestellten Sachverhalts durch (1) Erfassen der relevanten Information und (2) Interpretation ihrer Bedeutung.

Zum Erfassen der relevanten Information gehören die Vollständigkeit der erfassten Information (sofern diese relevant ist) und die Fähigkeit zur Unterscheidung von relevanten und irrelevanten (dekorativen) Bestandteilen einer Visualisierung. Inhaltliches Begreifen zeigt sich z. B. in der Unterscheidung zwischen ikonischen und symbolischen Darstellungen. Zur Interpretation gehört, dass eine oder mehrere Bedeutungen als weiterführende Gedanken aus einer Visualisierung entwickelt werden, gegebenenfalls unter Einbeziehung kultureller Normen. Weitere Beispiele zum Verstehen der Darstellung sind Erfassen der Gesamtbedeutung und kritisches Betrachten einer Darstellung im gegebenen Kontext.

3. „Verknüpfen multipler Darstellungen“ bezeichnet das Verbinden mehrerer Visualisierungen oder von Visualisierungen und Texten in Bezug auf Verstehen ihrer Beziehung sowie Erschließen des gemeinsamen Bedeutungsgehalts.

Es lassen sind zwei Teilaspekte hervorheben: (1) Text-Bild-Integration als Verknüpfen von Text und Visualisierungen und (2) Bild-Bild-Integration als Verknüpfen mehrerer Visualisierungen miteinander. Zu beiden Fällen gehört das Nachvollziehen von Repräsentationswechseln. Bei der Text-Bild-Integration enthält der Text zusätzliche Informationen, die in der Visualisierung nicht gegeben sind, und geht damit über eine reine Aufgabenstellung oder Anweisung hinaus. Zur Bild-BildIntegration gehören Erfassen von Gemeinsamkeiten und Unterschieden mehrerer Visualisierungen und Erkennen von Zusammenhängen. Dies manifestiert sich beispielsweise in zu sortierenden Einzeldarstellungen wie Ordnen von Körpern nach dem Volumen, Zuweisen von symbolischen und ikonischen Darstellungen in Mathematik oder logischem Anordnen von Bildern zu einer Bildergeschichte in Deutsch.

4. „Generieren von Darstellungen“ bezeichnet eigenständiges Erstellen geeigneter Visualisierungen sowie Vervollständigen bestehender Visualisierungen aufgrund bekannter oder gegebener Sachverhalte.

Darunter sind (1) Text-Bild-Transformation, (2) Bild-Bild-Transformation sowie (3) Erstellen von Visualisierungen aus dem Vorwissen heraus zu verstehen. Unter Text-Bild-Transformation wird visuelles Darstellen von Texten oder Textteilen, beispielsweise das Erstellen eines Clusters aus einem Sachtext in Deutsch oder die grafische Darstellung quantitativer Sachverhalte aus einem Text oder einer Tabel- 
le in Mathematik gefasst. Bild-Bild-Transformation wird häufig durch den Begriff Repräsentationswechsel erfasst, beispielsweise wenn in Mathematik zu einem Wassertank ein Graph mit der Höhe des Wasserspiegels zu zeichnen ist. Bei hoher Ausprägung der Kompetenzfacette gehört zum „Generieren von Darstellungen“ auch die eigene Entscheidung für die geeignete Darstellungsart. Während es sich bei Erstellung aus dem Vorwissen sowie Text-Bild-Transformation um rein produktive Komponenten handelt (da Textverstehen zwar rezeptiv ist, aber nicht zu Visualisierungskompetenz gehört), enthält die Bild-Bild-Transformation eine produktive ebenso wie rezeptive Komponente. In allen Fällen kann „Generieren von Darstellungen“" sowohl als Neuerstellung als auch als Vervollständigen bestehender visueller Darstellungen (aus Texten, Vorwissen oder anderen Darstellungen) geschehen.

Folgende Aspekte wurden im Verlauf der Expertendiskussion pointiert:

- Die Übergänge zwischen „Erkennen der Darstellung“ und ,Verstehen der Darstellung" sind in manchen Fällen fließend. Zur Unterscheidung kann das Modell zum „Entschlüsseln, Lesen und Interpretieren grafischer Darstellungen“ von Eichler und Vogel (2013) dienen, welches eine Unterscheidung in ,read-the-data', ,readin-the-data “ und ,read-beyond-the-data“ vornimmt. Dem „Erkennen der Darstellung“" werden im Strukturmodell all jene Aspekte zugeordnet, die Voraussetzung für ,read-the-data' sind, d. h. welche eine reine Kenntnisnahme bzw. Erkennen der Bestandteile der Visualisierung beinhalten. Da die Fähigkeit zu ,read-the-data ' bereits Verständnis für die Visualisierung verlangt, ist dieses in der vorliegenden Kompetenzmodellierung dem „Verstehen der Darstellung“ zugeordnet. Dabei geht es um Erfassen aller relevanten Informationen und um Interpretation der Darstellung als zwei Pole von ,read-in-the-data'. ,Read-beyond-the-data' ist nur dann im Strukturmodell beinhaltet, wenn ein klarer Bezug zur Visualisierung erkennbar ist - fehlt dieser oder geht er über den in der Visualisierung gezeigten Sachverhalt deutlich hinaus, ist dieser Aspekt nicht mehr Visualisierungskompetenz zuzurechnen und somit im Modell nicht enthalten.

- „Verstehen der Darstellung“ bezieht sich auf eine Visualisierung und „Verknüpfen multipler Darstellungen" auf Verstehen mehrerer Darstellungen in ihrer Gesamtheit. Die Unterscheidung, ob es sich im konkreten Fall um eine oder mehrere Darstellungen handelt, hängt davon ab, ob einzelne Bestandteile der Darstellungen separat betrachtet werden können und dann immer noch eine Bedeutung haben, welche der ursprünglichen Bedeutung in der kombinierten Darstellung nahekommt. Beispielsweise kann ein Koordinatensystem mit mehreren Geraden nicht ohne Bedeutungsverlust in zwei Darstellungen aufgeteilt werden, während dies bei zwei nebeneinanderstehenden Koordinatensystemen mit jeweils einer Gerade sehr wohl geht; ähnlich wie dies bei einzelnen Bildern einer Bildergeschichte möglich ist.

- „Generieren von Darstellungen“ erfasst visuelles Erstellen oder Verändern struktureller Inhalte (im Gegensatz zum Erinnern oder Reproduzieren zuvor betrachteter Darstellungen). Beispielsweise ist ein Markieren von in Darstellungen vorhandenen Strukturen (wie Einzeichnen eines Weges in einem Labyrinth oder Markieren eines Anteils bei einer Figur mit vorhandener Einteilung) kein Generieren, sondern vielmehr dem „Erkennen“ oder „Verstehen der Darstellung“ zuzuordnen. 
Dimensionalität des Konstrukts. Je nach Unterscheidung gemäß der Modalität oder der Facetten liegt dem Konstrukt Visualisierungskompetenz ein zwei- oder vierdimensionales Modell zugrunde:

- Zweidimensionalität: Gemäß der Rezeptivität/Produktivität kann Visualisierungskompetenz als zweidimensionales Konstrukt mit rezeptiver Komponente und produktiver Komponente (rein produktiv beim Generieren ohne visuelle Vorgaben) aufgefasst werden.

- Vierdimensionalität: Gemäß der Einteilung in die vier Kompetenzfacetten Erkennen, Verstehen, Verknüpfen und Generieren wird ein vierdimensionales Konstrukt beschrieben.

Abhängigkeiten und Beziehungen der Kompetenzfacetten. Die Expertenbefragung lässt vermuten, dass die Kompetenzfacetten (teilweise) aufeinander aufbauen. Jedoch lassen sich die Verhältnisse und Abhängigkeiten zwischen den Kompetenzfacetten durch die Expertenbefragung und Gruppendiskussion nicht eindeutig beantworten. So ist z. B. ungeklärt, ob „Generieren von Darstellungen“ das „Verknüpfen multipler Darstellungen“ beinhaltet oder beide Facetten unabhängige Kompetenzaspekte haben. Abb. 3 zeigt zwei mögliche Varianten: (a) Die vier Kompetenzfacetten sind aufeinander aufbauend, wobei „Generieren von Darstellungen“ die umfassendste Kompetenz ist. (b) „Verknüpfen multipler Darstellungen“ und „Generieren von Darstellungen“ basieren beide auf ,Verstehen der Darstellung“, enthalten jedoch jeweils voneinander unabhängige Kompetenzanteile.

Abbildung 3: Zwei mögliche Beziehungen der Kompetenzfacetten

a)

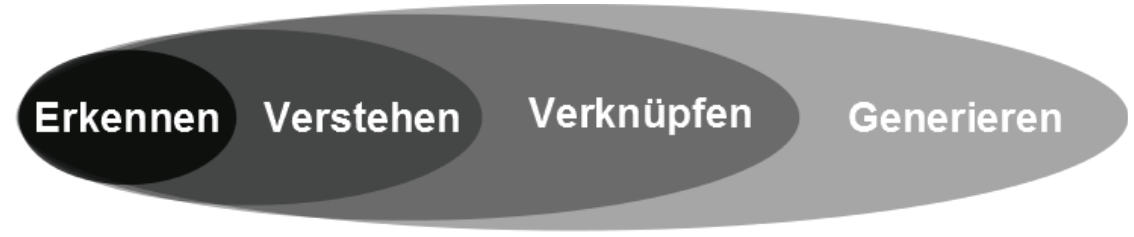

b)

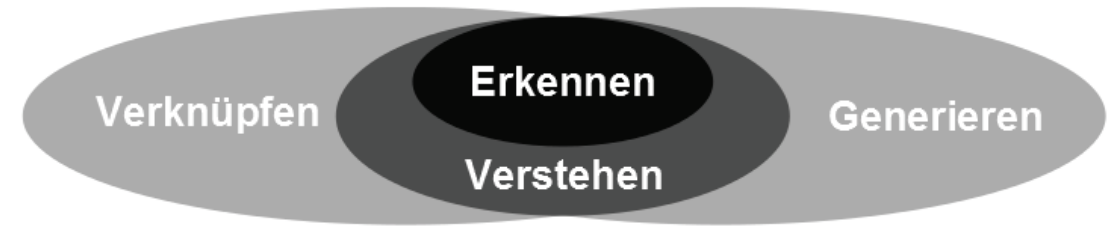

\section{Fazit und Ausblick}

Die qualitativen Untersuchungen zu Visualisierungskompetenz führten zur Entwicklung des in Abb. 2 dargestellten Kompetenzstrukturmodells. Durch die Expertenbefra- 
gung konnten deutliche Indizien gefunden werden, welche die Annahme eines mehrdimensionalen Kompetenzmodells nahelegen. Implizit wird durch die Betrachtungen postuliert, dass Visualisierungskompetenz eigenständige Kompetenzanteile hat, die sich von verwandten Konstrukten wie Abstraktem Denken, Lesekompetenz, Vorwissen, Intelligenz und Räumlichem Vorstellungsvermögen, abgrenzen lassen.

Durch die Explikation einzelner Kompetenzfacetten erlaubt das Strukturmodell in bestehender Form eine Sensibilisierung für die Thematik. Des Weiteren ermöglicht eine Fokussierung einzelner Aspekte Beiträge zur individuellen Förderung von Visualisierungskompetenz. So können in weiterer Forschungsarbeit zielgerichtete Aufgaben und Lernkontexte für Lehrpersonen entwickelt werden, die den Umgang mit Visualisierungen bei Schülerinnen und Schülern fachübergreifend schulen, um schließlich die ausgebildeten Kompetenzen auch fachspezifisch-förderlich anwenden zu können. Das Strukturmodell kann schließlich als Ausgangspunkt für die Entwicklung eines Diagnoseinstrumentariums für die pädagogische Praxis dienen. Es bietet die Basis einer Itemkonstruktionsheuristik und kann fachübergreifend sowie fachspezifisch konkretisiert werden.

Auf die eingangs aufgeworfene Frage, welche Kompetenzstruktur sich für den Umgang mit Visualisierungen in den Fächern Deutsch und Mathematik aus theoretischen und empirischen Erkenntnissen identifizieren lässt, zeigt dieser Artikel, welchen Beitrag die fachdidaktische qualitative Forschung im Prozess dieser Klärung zu leisten vermag. Als sehr ergiebig hat sich in methodischer Hinsicht die strukturierte sequentielle Anwendung einer literaturbasierten Identifikation und konsensuellen Kategorisierung mit der qualitativen Inhaltsanalyse leitfadengestützter Experteninterviews mit Strukturlegetechnik erwiesen. Insbesondere die konsequente Offenhaltung bei den Experteninterviews und das offene Kodieren stellten sich als zielbringend bezüglich der Dimensionalisierung des Kompetenzkonstrukts heraus.

Zugleich ist damit weiterer Forschungsbedarf markiert. Weitergehende qualitative Untersuchungen betreffen einerseits die Konsolidierung und weitere Präzisierung des entwickelten theoretischen Modells, wenn möglich auch eine Erweiterung von statischen auf dynamische Visualisierungen. Hierfür bieten sich Untersuchungen mit einer größeren Expertenanzahl anhand der Delphi-Methode (Häder \& Häder 2000) an. Verwandte Untersuchungen wurden im angloamerikanischen Raum durchgeführt (Baca \& Braden 1990, Brill et al. 2007), sind jedoch weder direkt auf den deutschsprachigen Lehr- und Lernkontext übertragbar noch auf das schulische Unterrichtsumfeld ausgerichtet. Da eine Übertragbarkeit von Forschungsergebnissen von anderen Kontexten und höheren Altersstufen auf die Sekundarstufe I und den schulischen Unterricht nicht gegeben sein muss (McTigue 2009), besteht hier weiterer Forschungsbedarf.

Schließlich sollte die präsentierte Expertenperspektive durch die Schülerperspektive erweitert werden, um die eigentliche Zielgruppe ins Zentrum der Untersuchung zu stellen. Es ist ein fundiertes Verständnis zu entwickeln, wie Schülerinnen und Schüler mit Visualisierungen umgehen und in ihrer Erfassung vorgehen. Dafür sind die ThinkAloud-Methode, Comprehension Probing sowie Unterrichts- und Schülerbeobachtungen geeignete qualitative Methoden, die im Umfeld von Visualisierungskompetenz für weitere Forschungserkenntnisse sorgen werden. 


\section{Autorenangaben}

Sammy Wafi, M.A.

Institut für Psychologie

Abteilung für Forschungsmethoden

Pädagogische Hochschule Freiburg

sammy.wafi@ph-freiburg.de
Prof. Dr. Markus Wirtz

Institut für Psychologie

Abteilung für Forschungsmethoden

Pädagogische Hochschule Freiburg

markus.wirtz@ph-freiburg.de

\section{Literatur}

Ainsworth, S. (1999): The functions of multiple representations. In: Computers \& Education 33, 2, S. 131-152.

Ainsworth, S. (2006): DeFT: A conceptual framework for considering learning with multiple representations. In: Learning and Instruction 16, 3, S. 183-198.

Anderson, J. R. (2010): Cognitive psychology and its implications. 7th ed. New York: Worth Publishers.

Avgerinou, M./Ericson, J. (1997): A Review of the Concept of Visual Literacy. In: British Journal of Educational Technology 28, 4, S. 280-291.

Avgerinou, M. D. (2001): Visual Literacy: Anatomy and Diagnosis. Dissertation. University of Bath.

Avgerinou, M. D. (2007): Towards a Visual Literacy Index. In: Journal of Visual Literacy 27, 1, S. 29-46.

Avgerinou, M. D./Pettersson, R. (2011): Toward a Cohesive Theory of Visual Literacy. In: Journal of Visual Literacy 30, 2, S. 1-19.

Baca, J. C./Braden, R. A. (1990): The Delphi Study: A Proposed Method for Resolving Visual Literacy Uncertainties. In: Braden, R. A./Beauchamp, D. G./Baca, J. C. (Hrsg.): Perceptions of Visual Literacy. University of Central Arkansas, Conway, AR: IVLA, Inc., S. 99-106.

Bortz, J./Döring, N. (2006): Forschungsmethoden und Evaluation. Für Human- und Sozialwissenschaftler. Heidelberg: Springer.

Brill, J. M./Kim, D./Branch, R. M. (2007): Visual Literacy Defined - The Results of a Delphi Study: Can IVLA (Operationally) Define Visual Literacy. In: Journal of Visual Literacy 27, 1, S. 47-60.

Calvert, G./Spence, C./Stein, B. E. (2004): The handbook of multisensory processes. Cambridge, Mass: MIT Press.

ChanLin, L.-J. (1998): Animation to teach students of different knowledge levels. In: Journal of Instructional Psychology 25, 3, S. 166-175.

Charters, E. (2003): The use of think-aloud methods in qualitative research. In: Brock Education Journal 12, 2, S. 68-82.

Debes, J. L. (1970): The Loom of Visual Literacy. In: Williams, C. M./Debes, J. L. (Hrsg.): Proceedings of the First National Conference on Visual Literacy. New York: Pitman Publishing Corporation, S. 1-16.

Doll, J./Rehfinger, A. (2012): Historische Forschungsstränge der Schulbuchforschung und aktuelle Beispiele empirischer Schulbuchwirkungsforschung. In: Doll, J./Frank, K./Fickermann D./ Schwippert, K. (Hrsg.): Schulbücher im Fokus: Nutzungen, Wirkungen und Evaluation. Münster: Waxmann Verlag, S. 19-41.

Dondis, D. A. (1973): A primer of visual literacy. Cambridge, Mass: MIT Press. 
Eichler, A./Vogel, M. (2013): Leitidee Daten und Zufall. Von konkreten Beispielen zur Didaktik der Stochastik. 2. Auflage. Wiesbaden: Springer Fachmedien.

Ericsson, K. A./Simon, H. A. (1980): Verbal reports as data. In: Psychological Review 87, 3, S. 215-251.

Flammer, A. (2009): Entwicklungstheorien. Psychologische Theorien der menschlichen Entwicklung. 4. Auflage. Bern: Huber.

Fischer-Dardai, A./Kojanitz, L. (2010): Textbooks analysis methods for the longitudinal study of textbook contents. In: Erdmann, E./Popp, S./Schumann, J. (Hrsg.): Analyzing textbooks: Methodological Issues. Schwalbach/Ts: Wochen, S. 47-65.

Fransecky, R. B. (1970): The Use of Visual Communication to Enrich and Extend „Traditional“ Verbal Language Learning. In: Williams, C. M./Debes, J. L. (Hrsg.): Proceedings of the First National Conference on Visual Literacy. New York: Pitman Publishing Corporation, S. 152157.

Fransecky, R. B./Debes, J. L. (1972): Visual Literacy: A Way to Learn - A Way to Teach. Washington D.C.: Association for Educational Communications and Technology.

Häder, M. (2010): Empirische Sozialforschung. Eine Einführung. 2. Auflage. Wiesbaden: Verlag für Sozialwissenschaften.

Häder, M./Häder, S. (2000): Die Delphi-Technik in den Sozialwissenschaften. Methodische Forschungen und innovative Anwendungen. Wiesbaden: Westdeutscher Verlag.

Hochpöchler, U./Schnotz, W./Rasch, T./Ullrich, M./Horz, H./McElvany, N./Baumert, J. (2013): Dynamics of mental model construction from text and graphics. In: European Journal of Psychology of Education 28, 4, S.1105-1126.

Jong, T. de/Ainsworth, S. et al. (1998): Acquiring knowledge in science and mathematics: the use of multiple representations in technology based learning environments. In: Someren, M.W. van (Hrsg.): Learning with multiple representations. Advances in learning and instruction series. Oxford: Elsevier Science, S. 9-41.

Kaput, J. J. (1987): Representation Systems and Mathematics. In: Claude, J. (Hrsg.): Problems of representation in teaching and learning math. Hillsdale, New Jersey: Lawrence Erlbaum Associates, S. 19-26.

Klieme, E./Maag-Merki, K./Hartig, J. (2007): Kompetenzbegriff und Bedeutung von Kompetenzen im Bildungswesen. In: Bundesministerium für Bildung und Forschung (Hrsg.): Möglichkeiten und Voraussetzungen technologiebasierter Kompetenzdiagnostik. Eine Expertise im Auftrag des BMBF. Bildungsforschung Band 20. Bonn, Berlin: BMBF, S. 5-15.

KMK (2004): Bildungsstandards für den Mittleren Schulabschluss. Beschluss vom 04.12.2003. Hg. v. Ständige Konferenz der Kultusminister der Länder. München: Wolters Kluwer.

Krapp, A./Weidenmann, B. (2006): Pädagogische Psychologie. 5. Auflage. Weinheim: Beltz PVU.

Kruse, J. (2014): Qualitative Interviewforschung. Ein integrativer Ansatz. Grundlagentexte Methoden. Weinheim: Beltz Juventa.

Legge, G. E./Gu, Y./Luebker, A. (1989): Efficiency of graphical perception. In: Perception \& Psychophysics 46, 4, S. 365-374.

Leuders, T. (2014): Modellierungen mathematischer Kompetenzen - Kriterien für eine Validitätsprüfung aus fachdidaktischer Sicht. In: Journal für Mathematikdidaktik 35, 1, S. 7-48.

Mayer, R. E. (2005): The Cambridge handbook of multimedia learning. Cambridge: Cambridge University Press.

Mayring, P. (2010): Qualitative Inhaltsanalyse. Grundlagen und Techniken. 11. Auflage. Weinheim: Beltz.

McKay, E. (1999): An investigation of text-based instructional materials enhanced with graphics. In: Educational Psychology 19, 3, S. 323-335.

McTigue, E. M. (2009): Does multimedia learning theory extend to middle-school students? In: Contemporary Educational Psychology 34, 2, S. 143-153. 
OECD (2004): Learning for Tomorrow's World. First Results from PISA 2003. Organisation for Economic Operation and Development.

Paivio, A. (1990): Mental representations. A dual coding approach. Oxford psychology series no. 9. New York, Oxford: Oxford University Press.

Paquin, R. L. (1999): The Competencies of Visual Literacy. In: Griffin, R. E./Gibbs, W. J./ Wiegmann, B. (Hrsg.): Visual Literacy in an Information Age. Selected readings of the IVLA Annual Conference. Penn State, PA, S. 245-248.

Ploetzner, R./Lowe, R./Schlag, S. (2013): A Systematic Characterization of Cognitive Techniques for Learning from Textual and Pictorial Representations. In: Journal of Education and Learning 2, 2, S. 78-95.

Prenzel, M./Walter, O./Frey, A. (2007): PISA misst Kompetenzen. Eine Replik auf Rindermann (2006): Was messen internationale Schulleistungsstudien? In: Psychologische Rundschau 58,2, S. $128-136$.

Presmeg, N. C. (2006): Research on visualization in learning and teaching mathematics. In: Gutiérrez, A./Boero P. (Hrsg.): Handbook of Research on the Psychology of Mathematics Education, S. 205-235.

Reinders, Heinz (2005): Qualitative Interviews mit Jugendlichen führen. Ein Leitfaden. München: Oldenbourg.

Schnotz, W. (2010): Visuelles Lernen. In: Rost, D. (Hrsg.): Handwörterbuch Pädagogische Psychologie. Weinheim: Beltz, S. 927-935.

Schnotz, W./Bannert, M. (2003): Construction and interference in learning from multiple representation. In: Learning and Instruction 13, 2, S. 141-156.

Seels, B. A. (1994): Visual Literacy: The Definition Problem. In: Moore, D. M./Dwyer, F. M. (Hrsg.): Visual literacy. A spectrum of visual learning. Englewood Cliffs, N.J: Educational Technology Publications, S. 97-112.

Smaldino, S. E./ Russell, J. D. (2005): Instructional Technology and Media for Learning. Unter Mitarbeit von Robert Heinich und Michael Molenda. 8. Auflage. Upper Saddle River, NJ (u.a.): Pearson.

Stokes, S. (2002): Visual Literacy in Teaching and Learning: A Literature Perspective. In: Electronic Journal for the Integration of Technology in Education 1, 1, S. 10-19.

van der Meij, J. (2007): Support for learning with multiple representations. Designing simulationbased learning environments. Enschede: PrintPartners Ipskamp.

van Meter, P. (2001): Drawing construction as a strategy for learning from text. In: Journal of Educational Psychology 93, 1, S. 129-140.

Verschaffel, L./Luwel, K./Torbeyns, J./Dooren, W. (2009): Conceptualizing, investigating, and enhancing adaptive expertise in elementary mathematics education. In: European Journal of Psychology of Education 24, 3, S. 335-359.

Wahl, D. (2006): Lernumgebungen erfolgreich gestalten. Vom trägen Wissen zum kompetenten Handeln. 2. Auflage. Bad Heilbrunn: Klinkhardt.

Watkins, J. K./Miller, E./Brubaker, D. (2004): The role of the visual image: What are students really learning from pictorial representations? In: Journal of Visual Literacy 24, 1, S. 23-40.

Weidenmann, B. (1991): Lernen mit Bildmedien. Psychologische und didaktische Grundlagen. Weinheim: Beltz.

Weidenmann, B. (1994): Lernen mit Bildmedien. Psychologische und didaktische Grundlagen. Beltz Weiterbildung, Band 1, 2. Auflage. Weinheim, Basel: Beltz.

Weinbrenner, P. (1995): Grundlagen und Methodenprobleme sozialwissenschaftlicher Schulbuchforschung. In: Olechowski, R. (Hrsg.): Schulbuchforschung. Frankfurt a. M.: Lang.

Wileman, R. E. (1993): Visual communicating. Englewood Cliffs, NJ.: Educational Technology Publications. 


\section{Anhang}

Tabelle 1: Auf der Expertenbefragung basierende Änderungsaspekte und Konsequenzen für das Kompetenzstrukturmodell

\begin{tabular}{|c|c|c|c|}
\hline & Aspekt & Umgang & Begründung / Bemerkung \\
\hline A1 & $\begin{array}{l}\text { „Assoziieren von Dar- } \\
\text { stellungen“ wurde als } \\
\text { Verknüpfen mit dem Vor- } \\
\text { wissen missverstanden/ } \\
\text { Kritisierung der Begriffs- } \\
\text { wahl }\end{array}$ & $\begin{array}{l}\text { Ersetzen durch „Verknüpfen } \\
\text { multipler Darstellungen“. } \\
\text { Definition als Fähigkeit zwischen } \\
\text { verschiedenen Darstellungs- } \\
\text { formen wechseln zu können. }\end{array}$ & $\begin{array}{l}\text { 7/14 der Expertinnen und } \\
\text { Experten schlug eine Begriffsän- } \\
\text { derung vor. }\end{array}$ \\
\hline B1 & $\begin{array}{l}\text { „Kenntnis des Darstel- } \\
\text { lungsformats“ und „Ver- } \\
\text { stehen der Darstellung“ } \\
\text { [alte Bezeichnung] hängen } \\
\text { zusammen. Die Unter- } \\
\text { scheidung ist fließend, die } \\
\text { Überlappungen sind groß. }\end{array}$ & $\begin{array}{l}\text { Aufteilung in Erkennen der Dar- } \\
\text { stellung, Erfassen der relevanten } \\
\text { Information und Interpretieren } \\
\text { der Darstellung in Strukturmodell } \\
\text { und Definitionen. Die Dreiteilung } \\
\text { besteht nur auf Konkretisie- } \\
\text { rungsebene; auf Facettenebene } \\
\text { wird „Erkennen“ und „Verste- } \\
\text { hen“ beibehalten. }\end{array}$ & $\begin{array}{l}\text { Argumente der Expertinnen und } \\
\text { Experten beim Strukturlegen } \\
\text { (9/14 haben „Kenntnis“ und } \\
\text { „Verstehen“ stärker aufge- } \\
\text { spalten) und bei der Think- } \\
\text { Aloud-Methode } \\
\text { (bei } 6 \text { Darstellungen aufgewor- } \\
\text { fen: } 3 \text { Mathematik, } 3 \text { Deutsch). }\end{array}$ \\
\hline B2 & $\begin{array}{l}\text {,Verständnis für die } \\
\text { Darstellungsart (ikonisch } \\
\text { vs. symbolisch)“ wurde } \\
\text { „Kenntnis des Darstel- } \\
\text { lungsformats“ oder „Ver- } \\
\text { stehen der Darstellung“" } \\
\text { zugeordnet }\end{array}$ & $\begin{array}{l}\text {,Verständnis für die Darstel- } \\
\text { lungsart (ikonisch vs. symbo- } \\
\text { lisch)‘ gehört zum „Verstehen } \\
\text { der Darstellung“ zwischen } \\
\text { „Erfassen der relevanten Infor- } \\
\text { mation“ (C1) und ,Interpretation } \\
\text { der Darstellung“. }\end{array}$ & $\begin{array}{l}\text { Die Mehrheit der Expertinnen } \\
\text { und Experten }(9 / 14) \text { hat diesen } \\
\text { Aspekt aufgeworfen. }\end{array}$ \\
\hline B3 & $\begin{array}{l}\text { Unterscheidung von rezep- } \\
\text { tiver/passiver und produk- } \\
\text { tiver/aktiver Komponente, } \\
\text { z. B. ,Bild-Bild-Transform- } \\
\text { ation' hat rezeptive sowie } \\
\text { produktive Komponenten; } \\
\text { 'Text-Bild-Transformation' } \\
\text { ist primär produktiv }\end{array}$ & $\begin{array}{l}\text { Hinweis auf Zweidimensiona- } \\
\text { lität: rezeptiv vs. produktiv, } \\
\text { Verankerung der Modalität im } \\
\text { Strukturmodell } \\
\text { - „Erkennen“, „Verstehen“ und } \\
\text { " „Verknüpfen“ sind rezeptiv } \\
\text { - „Generieren“ ist produktiv“ }\end{array}$ & $\begin{array}{l}\text { Aspekt wurde von } 4 \text { Expertinnen } \\
\text { und Experten aufgeworfen, auch } \\
\text { als Visuelles Lesen. }\end{array}$ \\
\hline B4 & $\begin{array}{l}\text { - ,Transformation“ hat } \\
\text { integrative Aspekte } \\
\text { - ,Text-Bild-“ und ,Bild- } \\
\text { Bild-Integration“ gehö- } \\
\text { ren zu „Verstehen der } \\
\text { Darstellung“ } \\
\text { - ,Bild-Bild-Transforma- } \\
\text { tion“ gehört zu „Kennt- } \\
\text { nis“ und „Assoziieren“ }\end{array}$ & $\begin{array}{l}\text { Zum Verhältnis der Facetten kann } \\
\text { durch die Expertenbefragung } \\
\text { nicht beantwortet werden, ob } \\
\text { „Generieren von Darstellungen“ } \\
\text { das „Verknüpfen multipler } \\
\text { Darstellungen“ beinhaltet oder } \\
\text { beide unabhängige Kompetenza- } \\
\text { spekte haben (Abb. 3). }\end{array}$ & $\begin{array}{l}\text { Die ursprünglichen Begrifflich- } \\
\text { keiten führten zu einzelnen } \\
\text { Missverständnissen und wurden } \\
\text { wie beschrieben überarbeitet. } \\
\end{array}$ \\
\hline C1 & $\begin{array}{l}\text { Neuer Aspekt: „Unter- } \\
\text { scheiden von wichtigen } \\
\text { und unwichtigen Teilen der } \\
\text { Darstellung“ / Erkennen } \\
\text { der Relevanz der Bestand- } \\
\text { teile einer Darstellung }\end{array}$ & $\begin{array}{l}\text {,Vollständiges Erfassen der } \\
\text { dargestellten Information“ wird } \\
\text { präzisiert durch ,Erfassen der } \\
\text { relevanten Information‘. } \\
\text { Wichtiger als reine Vollstän- } \\
\text { digkeit ist es, alle relevanten } \\
\text { Informationen aufzunehmen. }\end{array}$ & $\begin{array}{l}\text { Über die Hälfte der Expertinnen } \\
\text { und Experten ( } 8 / 14) \text { wiesen auf } \\
\text { diesen Punkt während des Struk- } \\
\text { turlegens hin, ebenso wie bei } 5 \\
\text { Darstellungen der Think-Aloud- } \\
\text { Methode ( } 3 \text { Mathe, } 2 \text { Deutsch). }\end{array}$ \\
\hline
\end{tabular}




\begin{tabular}{|c|c|c|c|}
\hline C2 & $\begin{array}{l}\text { Neue Konkretisierung: } \\
\text {,Bewerten von Visuali- } \\
\text { sierungen“ als Teil von } \\
\text { „Verstehen“: Wann ist } \\
\text { welche Darstellung wofür } \\
\text { geeignet? }\end{array}$ & $\begin{array}{l}\text { Entspricht einer hohen Aus- } \\
\text { prägung bei „Generieren von } \\
\text { Darstellungen“ und wird in die } \\
\text { Definition integriert. }\end{array}$ & $\begin{array}{l}\text { „Select an appropriate represen- } \\
\text { tation“ bei Ainsworth } 2006 \text {. }\end{array}$ \\
\hline D1 & $\begin{array}{l}\text {,Text-Bild-Integration“ hat } \\
\text { viel mit „Verstehen der } \\
\text { Darstellung“ zu tun }\end{array}$ & $\begin{array}{l}\text { Keine Anpassung im Strukturmo- } \\
\text { dell erforderlich. } \\
\text { Unterstützt die Facettenüber- } \\
\text { blicksdarstellung (Abb. 3) }\end{array}$ & $\begin{array}{l}\text { „Verknüpfen von Darstellungen“ } \\
\text { hat eine Verstehenskomponente, } \\
\text { d. h. „Verknüpfen“ ist Verstehen } \\
\text { mehrerer Darstellungen in ihrer } \\
\text { Gesamtheit. }\end{array}$ \\
\hline D2 & $\begin{array}{l}\text { Visualisierung ist nicht nur } \\
\text { Lernhilfe, sondern auch } \\
\text { Lerninhalt }\end{array}$ & $\begin{array}{l}\text { Hinweis für Definition und Kate- } \\
\text { gorisierung von Visualisierungen }\end{array}$ & $\begin{array}{l}\text { Bezieht sich auf die Seite der } \\
\text { Grafik und Funktion von Visuali- } \\
\text { sierungen, nicht auf die Seite der } \\
\text { Lernenden. }\end{array}$ \\
\hline E1 & $\begin{array}{l}\text { Neuer Aspekt: Visuelles } \\
\text { Erinnern } \\
\text { - Visuelles Langzeitge- } \\
\quad \text { dächtnis } \\
\text { - Visuelles Kurzzeitge- } \\
\quad \text { dächtnis } \\
\text { (Visual Memory bei Avgeri- } \\
\text { nou 2001) }\end{array}$ & $\begin{array}{l}\text { Keine Aufnahme von Visuellem } \\
\text { Erinnern als eigene Kompetenz- } \\
\text { facette } \\
\text { Die Komponente des visuellen } \\
\text { Kurzzeitgedächtnisses kann als } \\
\text { Operationalisierung für „Erken- } \\
\text { nen“ und „Verstehen“ eingesetzt } \\
\text { werden. Dies kann durch eine } \\
\text { statistische Tiefenanalyse auch } \\
\text { Indizien für die Bedeutung von } \\
\text { Visual Memory hervorbringen. }\end{array}$ & $\begin{array}{l}\text { Bedeutung für Visualisierungs- } \\
\text { kompetenz ist unklar (Avgeri- } \\
\text { nou 2001). Visuelles Erinnern } \\
\text { verlangt nach einem mentalen } \\
\text { Modell, da das Bild selbst nicht } \\
\text { mehr zur Verfügung steht. } \\
\text { Klare Abgrenzung zum „Gene- } \\
\text { rieren von Darstellungen“: Beim } \\
\text { Visuellen Erinnern wird eine } \\
\text { bestehende Visualisierung re- } \\
\text { produziert, beim Generieren wird } \\
\text { sie strukturell neu erschaffen. }\end{array}$ \\
\hline E2 & $\begin{array}{l}\text { Neuer Aspekt: Kritisches } \\
\text { Umgehen mit Visualisie- } \\
\text { rungen bzw. Beurteilen von } \\
\text { Visualisierungen: Was soll } \\
\text { damit erreicht werden? }\end{array}$ & $\begin{array}{l}\text { Keine explizite Abbildung im } \\
\text { Strukturmodell, jedoch mögliche } \\
\text { Integration bei „Verstehen der } \\
\text { Darstellung“. }\end{array}$ & $\begin{array}{l}\text { Entspricht Critical Viewing bei } \\
\text { Avgerinou (2001). }\end{array}$ \\
\hline E3 & $\begin{array}{l}\text { Unterscheidung von „Ver- } \\
\text { stehen der Darstellung“ in } \\
\text {,Zur Problemlösung“ oder } \\
\text {,Zum Verstehen der Struk- } \\
\text { tur eines Objekts“ }\end{array}$ & $\begin{array}{l}\text { Keine Abbildung dieser Unter- } \\
\text { scheidung im Strukturmodell }\end{array}$ & $\begin{array}{l}\text { Es handelt sich um eine } \\
\text { Unterscheidung nach Ziel bzw. } \\
\text { Prozess, nicht nach den Kompe- } \\
\text { tenzen auf Seiten der Lernenden. }\end{array}$ \\
\hline E4 & $\begin{array}{l}\text { Neuer Aspekt: Entwerfen } \\
\text { eigener Darstellungen } \\
\text { im Rahmen des Problem- } \\
\text { lösens }\end{array}$ & $\begin{array}{l}\text { Keine Abbildung im Struktur- } \\
\text { modell } \\
\text { Entsprechende Verdeutlichung } \\
\text { in der Definition von „Generieren } \\
\text { von Darstellungen“ }\end{array}$ & $\begin{array}{l}\text { Keine Visualisierungskompetenz, } \\
\text { sondern Problemlösefähigkeit/- } \\
\text { strategie; vorhandene Visuali- } \\
\text { sierungskompetenz kann dies } \\
\text { jedoch unterstützen. }\end{array}$ \\
\hline E5 & $\begin{array}{l}\text { Neuer Aspekt: Ästhetik / } \\
\text { gute Layouts / Umgehen } \\
\text { mit Farbe / Zeichenkom- } \\
\text { petenz }\end{array}$ & $\begin{array}{l}\text { Keine Abbildung im Struktur- } \\
\text { modell } \\
\text { Entsprechende Verdeutlichung } \\
\text { in der Definition von „Generieren } \\
\text { von Darstellungen“ }\end{array}$ & $\begin{array}{l}\text { Künstlerisch-ästhetische Kompe- } \\
\text { tenz steht fachlich bedingt nicht } \\
\text { im Zentrum der Untersuchung. }\end{array}$ \\
\hline
\end{tabular}

